\title{
MicroRNA-1908-5p contributes to the oncogenic function of the splicing factor SRSF3
}

\author{
Hye Ree Kim ${ }^{1}$, Chang Hoon Shin ${ }^{1}$, Hong Lee ${ }^{1}$, Kyung Hee Choi ${ }^{1}$, Do-Hyun Nam $^{1,2}$, \\ Takbum Ohn ${ }^{3}$, Hyeon Ho Kim ${ }^{1,4}$ \\ ${ }^{1}$ Department of Health Sciences and Technology, Samsung Advanced Institute for Health Sciences and Technology, \\ Sungkyunkwan University, Seoul, Republic of Korea \\ ${ }^{2}$ Department of Neurosurgery, Samsung Medical Center, Sungkyunkwan University School of Medicine, Seoul, Republic of \\ Korea \\ ${ }^{3}$ Department of Cellular and Molecular Medicine, College of Medicine, Chosun University, Gwangju, Republic of Korea \\ ${ }^{4}$ Research Institute for Future Medicine, Samsung Medical Center, Seoul, Republic of Korea \\ Correspondence to: Hyeon Ho Kim, email: hyeonhkim@skku.edu \\ Takbum Ohn, email: tohn@chosun.ac.kr \\ Keywords: SRSF3, FADS1, miR-1908-5p, NF-KB, NKIRAS2
}

Received: August 24, $2016 \quad$ Accepted: November 22, $2016 \quad$ Published: December 26, 2016

\section{ABSTRACT}

Serine/arginine (SR)-rich proteins that contain RS domains and SR repeats have diverse cellular functions including transcription, polyadenylation, translation, and RNA export. The splicing factor SRSF3, also termed SRp20, is the smallest member of the SR protein family and is a known proto-oncogene. Although it is implicated in the malignant phenotypes of various cancer cells, the molecular mechanism underlying SRSF3-mediated cancer progression is still obscure. We investigated here the oncogenic functions of SRSF3 in osteosarcoma U2OS cells. Knockdown of SRSF3 inhibited proliferation, clonogenicity, and metastatic potential including migration and invasion. It also decreased the level of miR-1908 independent of its host gene FADS1. Although FADS1 was not associated with SRSF3-mediated malignant properties, overexpression of $\mathrm{miR}-1908-5 p$ increased cell proliferation, migration, and invasion, suggesting that $\mathbf{m i R - 1 9 0 8 - 5 p ~ i s ~ r e s p o n s i b l e ~ f o r ~ t h e ~ o n c o g e n i c ~ f u n c t i o n s ~ o f ~}$ SRSF3. Knockdown of SRSF3 decreased the expression of miR-1908-5p by inhibiting transactivation of NF-KB. We observed that miR-1908-5p downregulated NF-KB inhibitor interacting Ras-like 2 (NKIRAS2), a negative regulator of the NF-KB pathway by directly binding to the 3'UTR of NKIRAS2 mRNA. Consistent with overexpression of miR-1908-5p, knockdown of NKIRAS2 diminished the expression level of IKB- $\beta$ and provoked translocation of NF-KB into the nucleus where it transcriptionally activates its target genes including miR-1908-5p expression, thus elevating the proliferation and metastatic potential. Taken together, our results demonstrate that SRSF3 confers the malignant characteristics on cancer cells via the SRSF3/miR-1908-5p/ NKIRAS2 axis.

\section{INTRODUCTION}

Serine/arginine (SR)-rich proteins containing at least one RNA recognition motif (RRM) and one arginine/ serine repeat (RS domain) have diverse cellular functions including transcription, polyadenylation, translation, and RNA export [1]. After SF2/ASF was identified as the first SR protein, others were subsequently classified on the basis of their molecular weight: SRp20, SRp40,
SRp55, and SRp75 [2]. Analyses based on cross-linking and immunoprecipitation coupled with high-throughput sequencing (iCLIP-seq) determine that SR proteins are able to bind to functionally diverse mRNAs including noncoding RNAs, suggesting that disruption of their functions may cause various diseases [3]. As a critical regulator of RNA metabolism, SR proteins have received attention from oncologists. Several reports have revealed that SR proteins play critical roles in cancer progression [4]. 
SRSF3 (SR-rich splicing factor 3, also termed SRp20) is the smallest member of the SR protein family and is known proto-oncogene [4, 5]. However, the oncogenic roles of SRSF3 remain largely unknown. SRSF3 is frequently upregulated in most types of cancer and is closely associated with the prognosis of cancer patients. In various systems, SRSF3 confers malignant phenotypes including increased proliferation, antiapoptosis, and gain of metastatic abilities (migration and invasion) [6-8]. SRSF3 has been recently reported to regulate alternative splicing and gene expression of forkhead box M1 (FoxM1), polo-like kinase 1 (PLK1), and cell division cycle 25B (Cdc25B) in U2OS osteosarcoma cells [4]. SRSF3 also translationally suppresses the expression of programmed cell death 4 (PDCD4) mRNA through an interaction with 5' untranslated region (UTR) of its mRNA [9].

MicroRNAs (miRNAs) are an example of many regulatory non-coding RNAs and are 19 25 nucleotides in length. They play an important role in the regulation of gene expression through translational suppression or mRNA degradation mainly by targeting 3' UTR of target mRNA [10]. Depending on their target genes, miRNAs are subdivided into oncogenic and tumor-suppressing miRNAs which suppress the expression of tumor suppressors and oncogenes, respectively. Accordingly, miRNAs widely influence cancer development and progression such as proliferation, anti-apoptosis, resistance, migration, and invasion [11]. For most miRNAs, primary miRNAs (primiRNAs) are transcribed by RNA polymerase II, either as separated or embedded transcripts. Although few miRNAs are known to have their own transcription factors, the majority of miRNAs are transcribed by transcription factors for their host genes.

In this study, we investigated the oncogenic function of SRSF3 in osteosarcoma U2OS cells. Our data reveals that SRSF3 confers malignant phenotypes such as increased proliferation, migration, and invasion on U2OS cells by upregulating NF- $\mathrm{KB}$-dependent oncogenic miR-1908-5p expression. We also found that the NF$\kappa \mathrm{B}$ inhibitor interacting Ras-like 2 (NKIRAS2) gene is a novel target of miR-1908-5p and is responsible for the reinforcement of the $\mathrm{NF}-\kappa \mathrm{B}$ pathway by $\mathrm{SRSF} 3 / \mathrm{miR}$ 1908-5p/NKIRAS2.

\section{RESULTS}

\section{Knockdown of SRSF3 inhibits clonogenicity and metastatic potential of $\mathrm{U} 2 \mathrm{OS}$ cells}

Growing evidences demonstrate that several SR proteins including SRSF1 [12], SRSF3 [4], and SRSF6 [13, 14] are classified into oncogenes that are involved in rapid proliferation and metastatic properties. We investigated the effect of SRSF3 silencing on clonogenicity and metastatic potential of osteosarcoma
U2OS cells. Short interference RNA (siRNA) was used to specifically knockdown SRSF3 expression. siRNAs targeting the coding region or 3'UTR of SRSF3 mRNA efficiently decreased the expression of SRSF3 (Figure 1A and Supplementary Figure 1A). SRSF3-knockdown U2OS cells exhibited reduced proliferation as compared to the control siRNA-transfected cells (Figure 1B). We also found that reduced expression of SRSF3 inhibited clonogenicity (Figure 1C and Supplementary Figure 1B) and suppressed metastatic abilities including invasion (Figure 1D, left panel and Supplementary Figure 1C) and migration (Figure 1D, right panel and Supplementary Figure 1D), indicating that SRSF3 is responsible for the malignant phenotypes of osteosarcoma U2OS cells.

\section{SRSF3-mediated regulation of $\mathrm{miR}-1908$ is independent of its host gene FADS1}

A list of differentially expressed genes by the knockdown of SRSF3 was previously reported [9]. Based on this, we hypothesized that SRSF3 is able to control the expression of miRNAs and screened SRSF3targeted genes harboring primary sequences of miRNAs in their introns. Among the 444 downregulated and 579 upregulated genes, only 40 genes (21 downregulated and 19 upregulated genes) harbor the primary sequences of miRNA (Supplementary Figure 2). Fatty acid desaturase 1 (FADS1) is one of the SRSF3 target genes and that contains the primary sequence of miR-1908 (primiR-1908) in its intron 1. By analyzing the microarray data, we found that FADS1 mRNA was decreased in SRSF3-silenced U2OS cells (Figure 2A). For validation, U2OS cells were transfected with SRSF3-specific siRNA and the level of FADS1 mRNA was assessed by RT-qPCR. Knockdown of SRSF3 significantly decreased the level of FADS1 mRNA (Figure 2B and Supplementary Figure 3A). It also decreased the level of pri-miR-1908 (Figure 2C and Supplementary Figure 3B) and miR-1908-3p and miR-1908-5p, two strands of its mature form as well (Figure 2D and Supplementary Figure 3C). These results indicated that SRSF3 is able to regulate the expression of miR-1908 and its host gene FADS1. To check whether $F A D S 1$ is involved in the decreased expression of miR1908 by the SRSF3 knockdown, we tested the level of miR-1908 in FADS1-silenced cells (Figure 2E). It was observed that the expression of miR-1908 was not affected by FADS1. Most intronic miRNAs are usually regulated by transcription factor of their host genes and as previously found, SRSF3 regulates the expression of various transcription factors such as Sp1, which is predicted as a putative transcriptional factor of FADS1 [15]. Given the marked decrease of Sp1 in SRSF3-silenced cells, we tested whether Sp1 is involved in the SRSF3mediated regulation of miR-1908. Interestingly, we found that knockdown of Sp1 did not affect the expression of miR-1908 although FADS1 mRNA was decreased in Sp1- 
silenced cells (Figure 2F and Supplementary Figure 3D). These results revealed that SRSF3 regulates the expression of miR-1908 independently from its host gene FADS1, suggesting that miR-1908 could be affected by its own transcription factors.

\section{FADS1 is not involved in the oncogenic function of SRSF3}

Since the knockdown of SRSF3 was found to decrease the expression level of FADS1, we investigated whether downregulation of FADS1 is required for the inhibitory effects of SRSF3 silencing. Designed FADS1specific siRNA effectively suppressed the expression of FADS1 (Supplementary Figure 4A). Unexpectedly, knockdown of FADS1 did not influence proliferation and clonogenicity of U2OS cells (Supplementary Figure $4 \mathrm{~B}$ and $4 \mathrm{C}$, respectively). Furthermore, the metastatic potential such as invasive and migratory abilities was not affected in FADS1-silenced cells (Supplementary Figure $4 \mathrm{D}$ and $4 \mathrm{E}$, respectively). These results indicated that FADS1 is not associated with the oncogenic function of SRSF3.

\section{miR-1908-5p confers malignant properties on U2OS cells}

Previously, we found that SRSF3 contributes to the malignant properties of $\mathrm{U} 2 \mathrm{OS}$ cells and FADS1, one of its target genes, is not responsible for the oncogenic function of SRSF3. Therefore, we checked whether miR-1908 is required for SRSF3-mediated malignancy. Although SRSF3 regulated the expression of both mature miRNAs, we further studied the roles of miR-1908-5p because its expression was more affected by the SRSF3 knockdown than its sister miRNA, miR1908-3p. To examine the effects of miR-1908-5p on cellular proliferation, U2OS cells were transfected with $S R S F 3$-specific siRNA and the proliferation rate was determined by counting viable cells every $24 \mathrm{~h}$. U2OS cells highly expressing miR-1908-5p showed an increased proliferation rate compared to parental cells (Figure 3A). In addition to proliferation, we also checked the effect of miR-1908-5p on metastatic properties. The invasive and migratory abilities were strengthened by overexpression of miR-1908-5p (Figure 3B and 3C). From the above results, we demonstrated that miR-1908-5p is closely associated with the oncogenic functions of SRSF3.
A
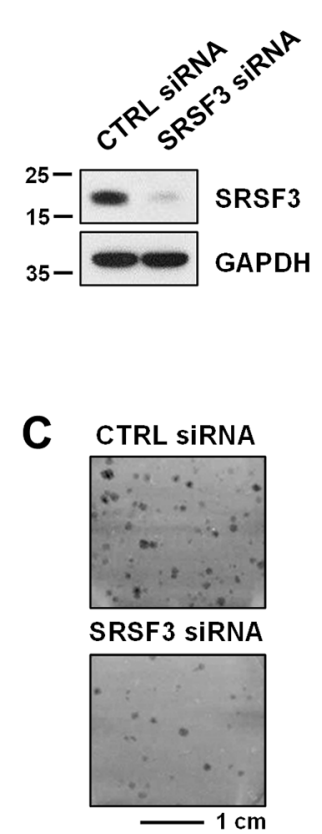

B
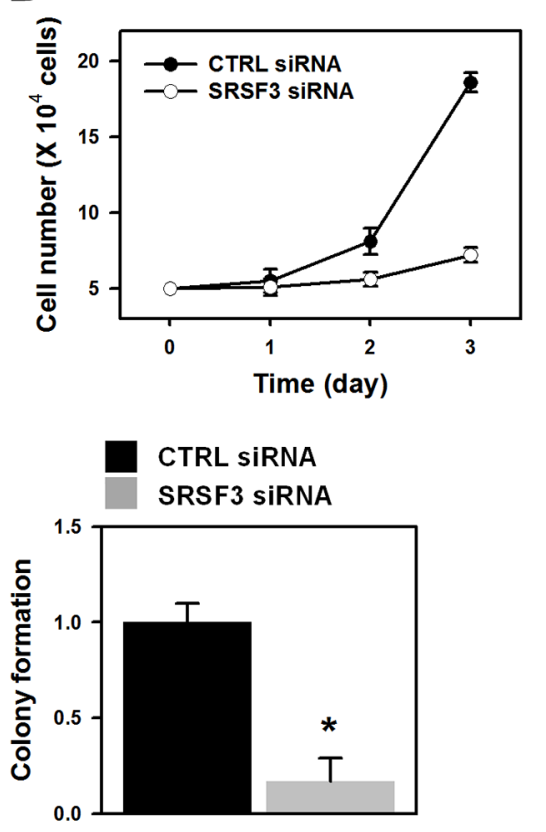

D
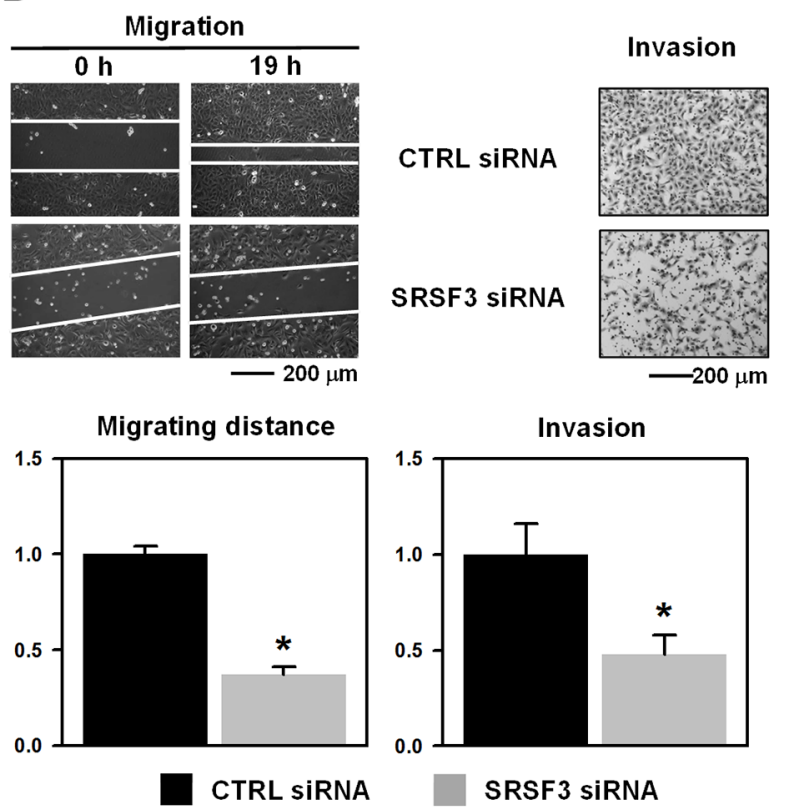

Figure 1: SRSF3 contributes to the malignant properties of U2OS cells. A. U2OS cells were transfected with control (CTRL) or SRSF3-specific siRNA. The expression level of SRSF3 was determined by western blot analysis and GAPDH was used as a loading control. B. The equal number of transfected cells was resuspended into 12-well plates and cellular proliferation was assessed by counting the number of viable cells at every $24 \mathrm{~h}$. C. For the clonogenic assay, transfected cells were plated into 6-well plates and cultured for more than 2 weeks. Clonogenic activity was assessed by counting the number of colonies. D. The migratory and invasive abilities were assessed by a wound healing assay and Matrigel invasion assay, respectively, as described in Materials and Methods. All experiments are performed more than three times and data represent the mean \pm S.D. Asterisk $\left(^{*}\right)$ indicates statistical significance of $p<0.05$ as determined by Student's t-test. 
The NF-KB pathway is implicated in the upregulation of miR-1908 by SRSF3

We realized that SRSF3-mediated regulation of miR1908 is unconnected with the expression of its host gene FADS1; therefore, we sought the molecular mechanism underlying how SRSF3 regulates miR-1908 expression. $\mathrm{NF}-\kappa \mathrm{B}$ has recently been identified as a transcriptional factor of miR-1908 [16]. Bioinformatics analysis revealed two putative NF- $\mathrm{kB}$ binding sites in the $1243 \mathrm{bp} \mathrm{miR-}$ 1908 promoter located in the intron of FADS1 [16]. With this in mind, we tested whether SFSR3 can activate the NF- $\kappa B$ pathway. First, we checked the effect of SRSF3 silencing on the transcriptional activity of NF- $\mathrm{KB}$ using a reporter vector (Figure 4A). Transactivation of NF- $\mathrm{KB}$ was diminished in $S R S F 3$-silenced cells. Secondary, we
A

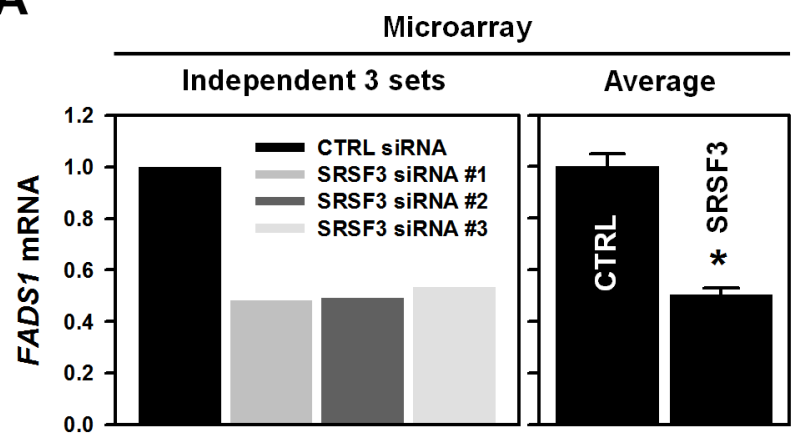

B

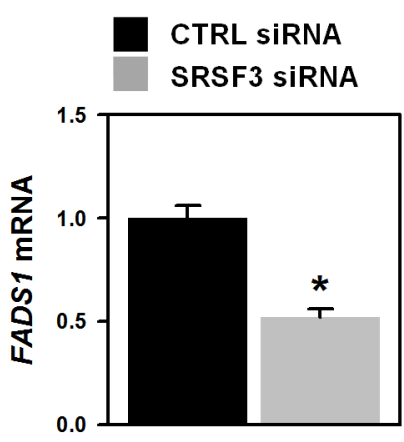

C

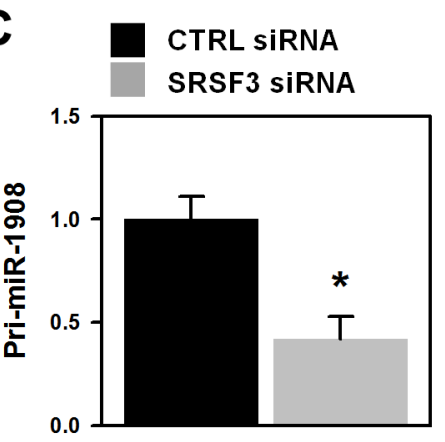

E

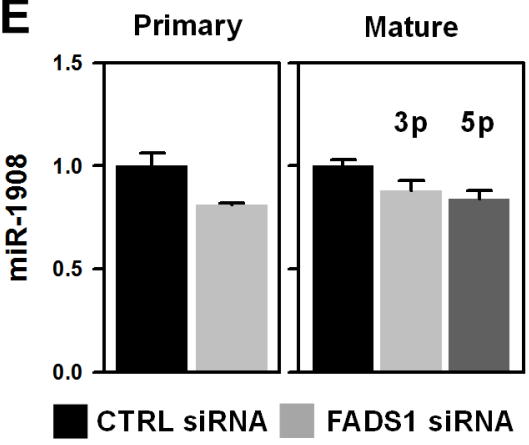

D

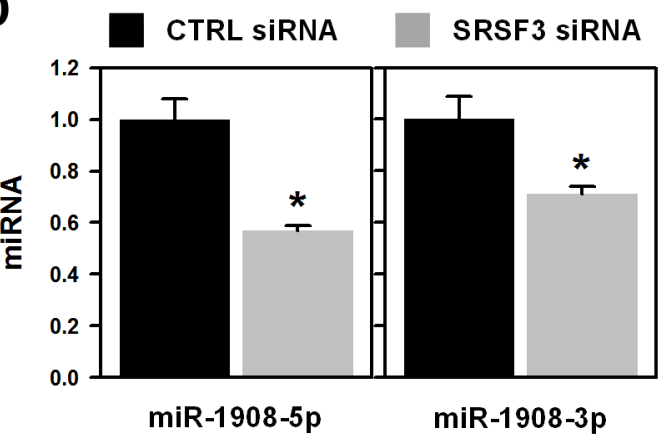

$\mathbf{F}$
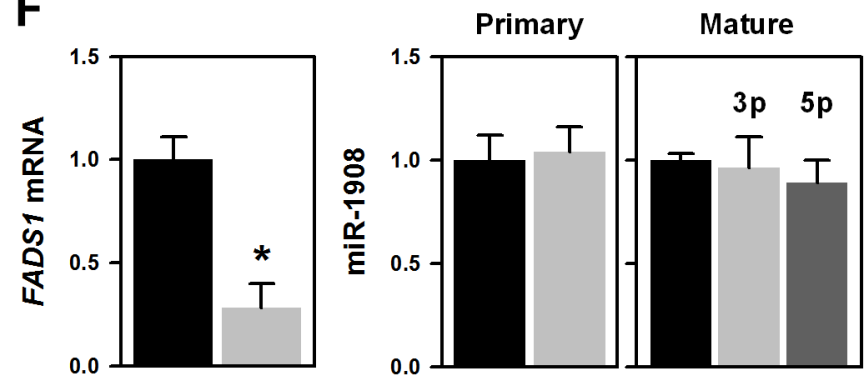

CTRL SIRNA Sp1 SIRNA

Figure 2: Knockdown of SRSF3 decreased the expression of miR-1908 and its host gene, FADS1. A. Microarray data showing decreased expression of FADS1 in SRSF3-silenced U2OS cells with three different siRNAs. B. U2OS cells were transfected with control (CTRL) or SRSF3-specific siRNA for $48 \mathrm{~h}$ and then the expression level of FADS1 mRNA was determined by RT-qPCR analysis. C-D. The levels of pri-miR-1908 and mature miR-1908-3p/5p were assessed by RT-qPCR analysis using pri-miR-1908-specific primers and TaqMan miRNA primers, respectively. E. Cells were transfected with control (CTRL) or FADS1-specific siRNA for $48 \mathrm{~h}$ and then the levels of pri-miR-1908 and mature miR-1908-3p/5p were assessed by RT-qPCR as described above. F. Cells were transfected with control (CTRL) or Sp1-specific siRNA for $48 \mathrm{~h}$. The expression level of FADS1 mRNA was determined by RT-qPCR analysis and the levels of pri-miR-1908 and mature miR-1908 (3p/5p) were assessed by RT-qPCR analysis as described above. All experiments are performed more than three times and data represent the mean \pm S.D. Asterisk $\left(^{*}\right)$ indicates statistical significance of $p<0.05$ as determined by Student's t-test. 
tested whether NF- $\kappa \mathrm{B}$ is implicated in SRSF3-regulated miR-1908 expression. U2OS cells were treated with the $\mathrm{NF}-\kappa \mathrm{B}$ inhibitor BAY 11-7082 for $24 \mathrm{~h}$ and then the levels of pri-miR-1908 and miR-1908-3p/5p were assessed by RT-qPCR. As shown in Figure $4 \mathrm{~B}$, the expressions of primary (left panel) and mature form (right panel) of miR-1908 were decreased by the inhibition of $N F-\kappa B$, revealing that SRSF3 regulates the expression of miR1908 through the NF- $\mathrm{BB}$ pathway. By investigating the molecular mechanism underlying the roles of SRSF3 in the NF- $\kappa$ B pathway, we found that SRSF3 is able to affect NF- $\kappa \mathrm{B}$ transactivation through transforming growth factor- $\beta$-activated kinase 1 (TAK1). TAK1 is known to regulate $\mathrm{NF}-\kappa \mathrm{B}$ transactivation through IKK $\alpha / \beta$-mediated phosphorylation of NF- $\mathrm{B}$ (p65) [17]. Knockdown of SRSF3 decreased the expression of TAK1 and thus suppressed the phosphorylation of IKK $\alpha / \beta$ and NF- $\mathrm{BB}$ (p65 subunit), indicating that SRSF3 is responsible for the activation of the NF- $\mathrm{B}$ pathway (Figure 4C). To verify this SRSF3-dependent activation, we checked the cytoplasmic and nuclear localization of $N F-\kappa B$ in SRSF3-silenced cells (Figure 4D). Knockdown of SRSF3 increased the level of cytoplasmic localization; however, the level of nuclear NF- $\mathrm{BB}$ (p65) was decreased in SRSF3silenced cells, indicating that SRSF3 activates the NF- $\kappa B$ pathway by triggering the nuclear localization of NF- $\kappa B$ (p65). To test whether TAK1 is responsible for SRSF3mediated NF- $\kappa \mathrm{B}$ activation, U2OS cells were transfected with control (CTRL) or TAK1-specific siRNA for $48 \mathrm{~h}$ and then the phosphorylated levels of IKK $\alpha / \beta$ and NF- $\kappa B$ (p65) were assessed (Figure 4E). Knockdown of TAK1 inhibited the phosphorylation of IKK $\alpha / \beta$ and $N F-\kappa B$. These results indicate that SRSF3 is able to positively influence the NF- $\mathrm{B}$ pathway by regulating the expression

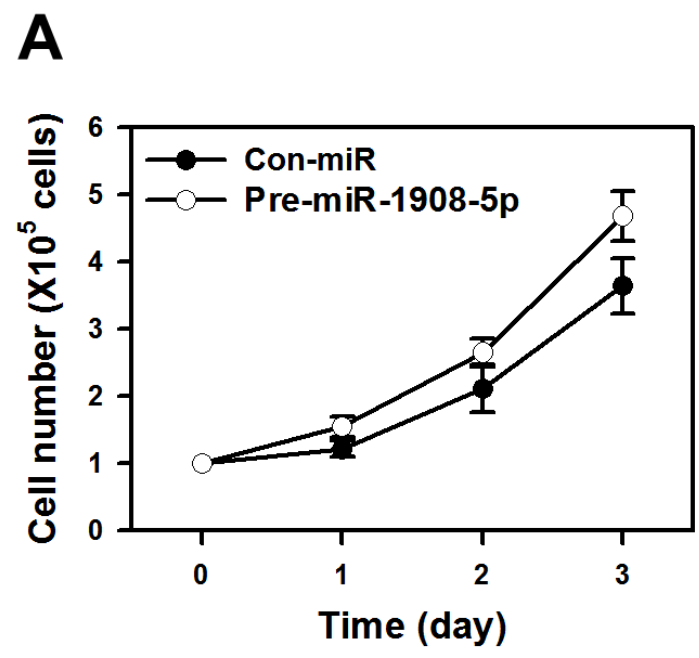

B

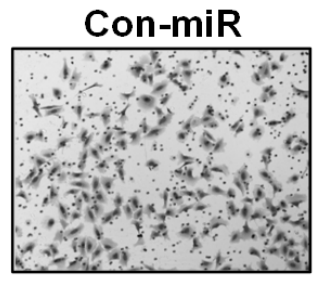

Pre-miR-1908-5p

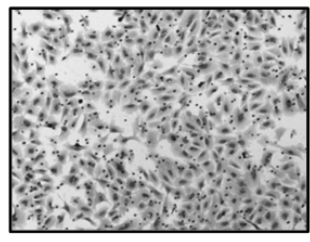

$18 \mathrm{~h}$

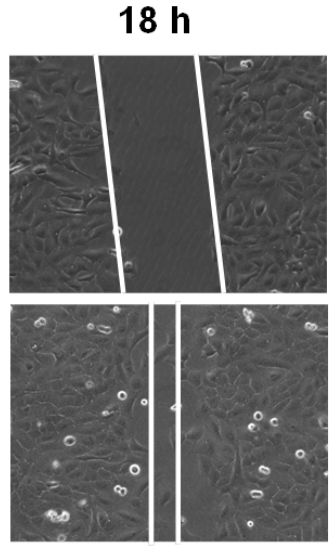

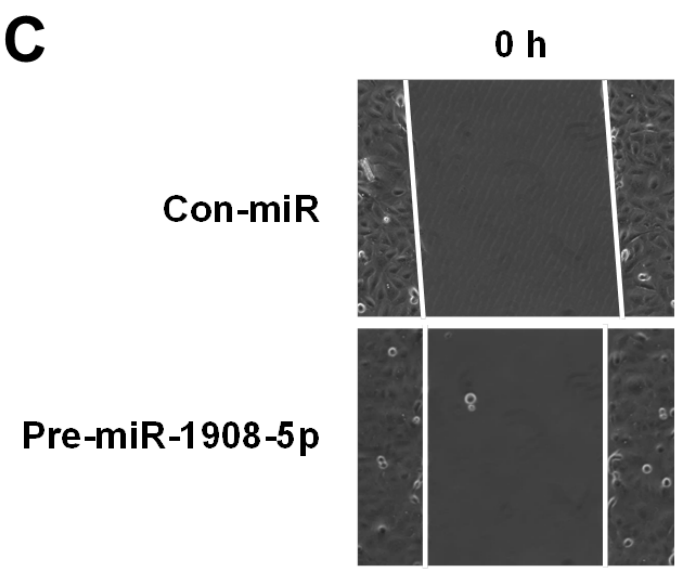
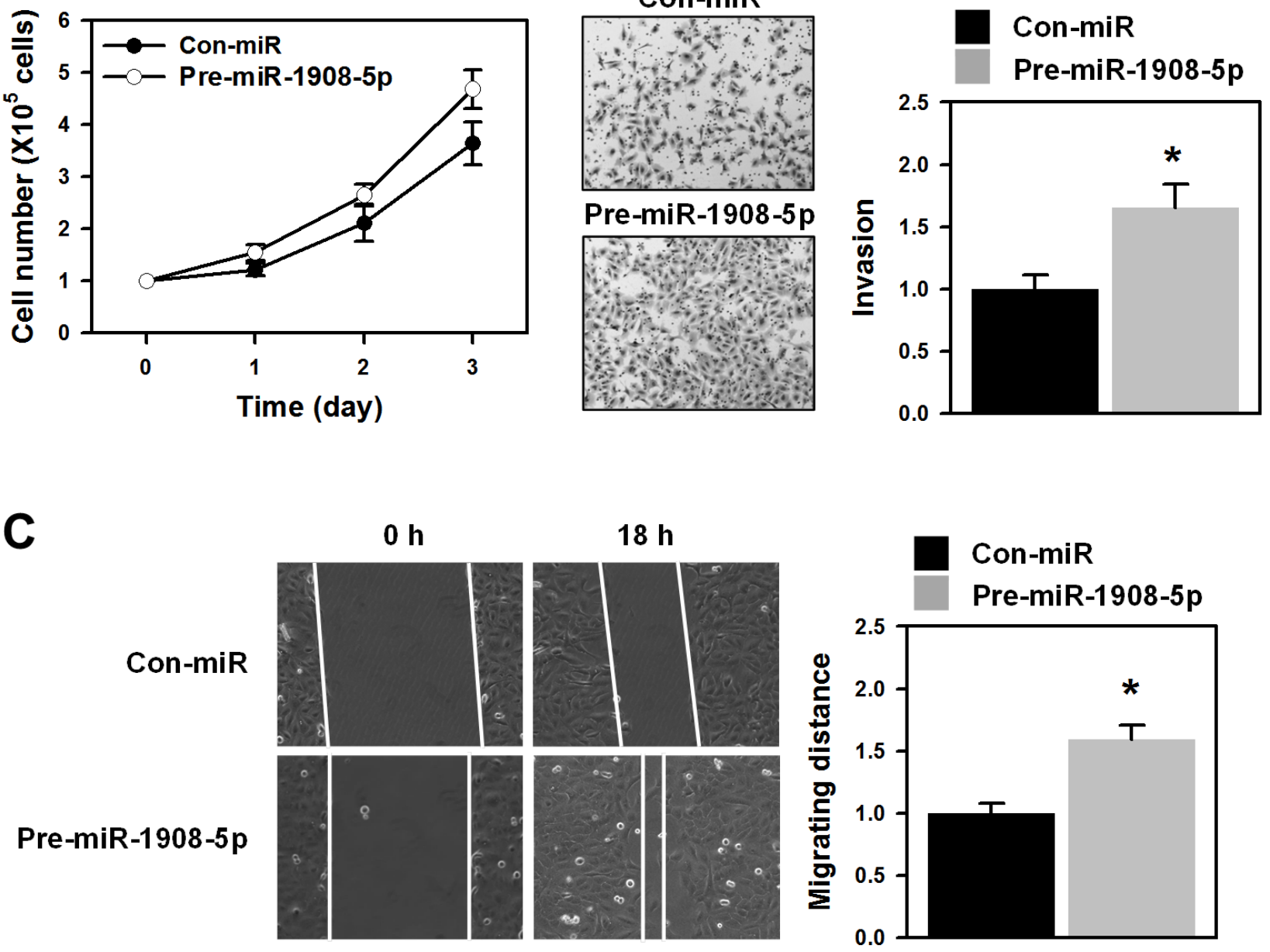

Figure 3: miR-1908-5p increased proliferation and metastatic potential. A. U2OS cells were transfected with control (Con-miR) or pre-miR-1908-5p for $24 \mathrm{~h}$ and then resuspended into 12-well plates. The proliferation rate was determined by counting the number of viable cells at every $24 \mathrm{~h}$. B-C. The invasive (B) and migratory (C) activities of miR-1908-5p-overexpressed cells were assessed by a wound healing assay and Matrigel invasion assay, respectively. All experiments are performed more than three times and data represent the mean \pm S.D. Asterisk $(*)$ indicates statistical significance of $\mathrm{p}<0.05$ as determined by Student's t-test. 
of TAK1. We also checked the level of miR-1908-5p and $\mathrm{NF}-\kappa \mathrm{B}$ target genes in TAK1-silenced cells. Knockdown of TAK1 decreased the expression level of miR-1908-5p
(Figure 4F). It also showed decreased protein and mRNA levels of NF- $\kappa$ B target genes (Figures $4 \mathrm{G}$ and $4 \mathrm{H}$, respectively).

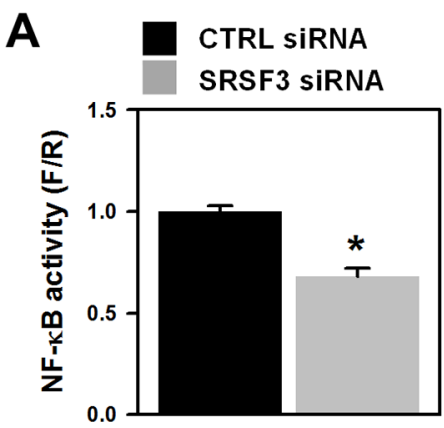

B

$0 \mu \mathrm{M}$ BAY 11-7082, $24 \mathrm{~h}$

$10 \mu \mathrm{M}$ BAY 11-7082, $24 \mathrm{~h}$
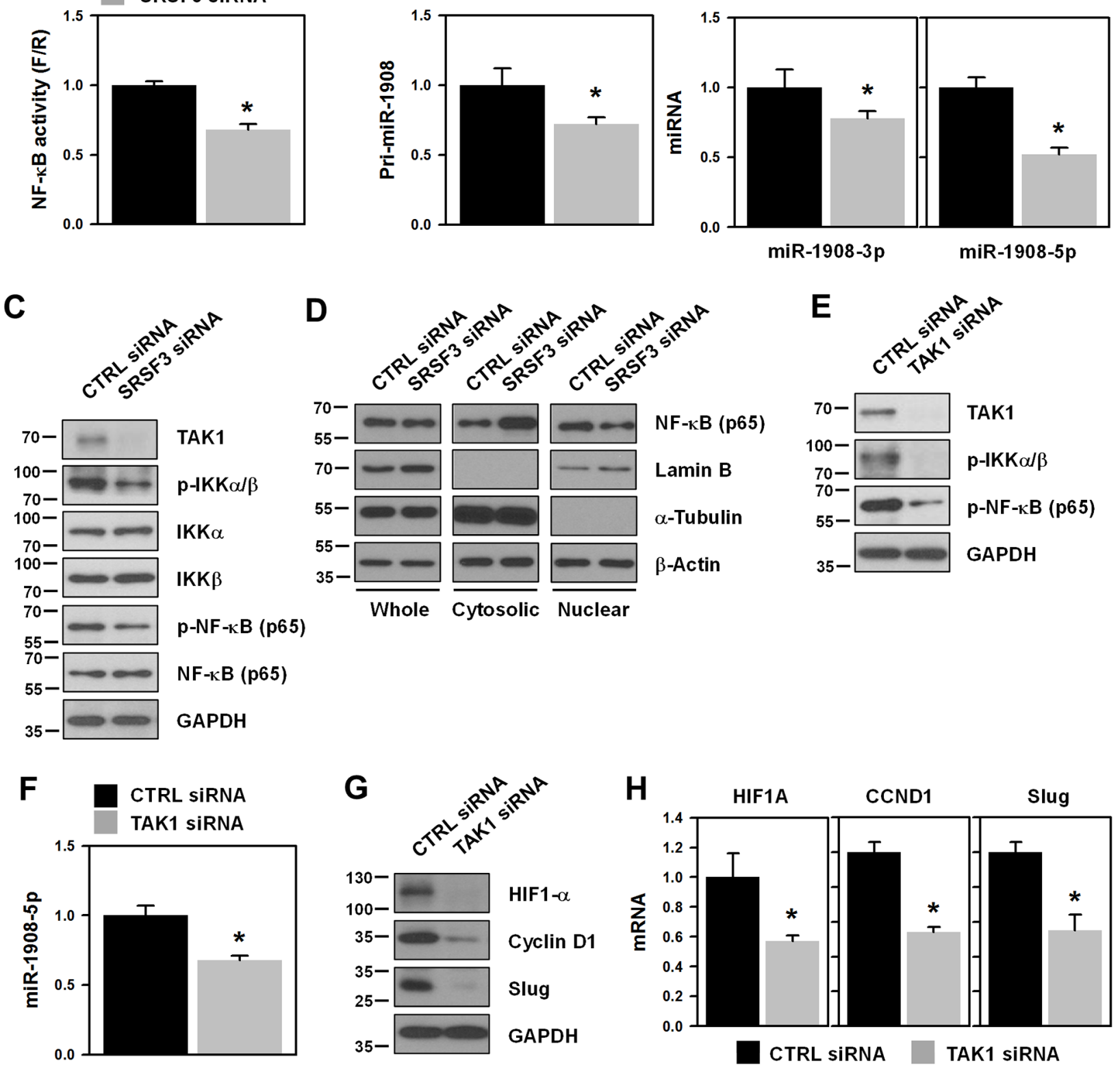

Figure 4: NF-кB is involved in SRSF3-regulated miR-1908 expression. A. U2OS cells were transfected with control (CTRL) or SRSF3-specific siRNA. Transfected cells were resuspended into 12-well plates and then transfected with the NF- $\mathrm{B}$ reporter vector. Transactivation of NF- $\kappa \mathrm{B}$ was assessed by calculating the luciferase activity. B. To check whether NF- $\mathrm{B}$ regulates the expression of miR1908, cells were treated with the NF-kB-specific inhibitor BAY11-7082 $(10 \mu \mathrm{M})$ for $24 \mathrm{~h}$. The level of miR-1908-3p/5p was determined by RT-qPCR analysis using TaqMan primers. C. Cells were transfected with control (CTRL) or SRSF3-specific siRNA for $48 \mathrm{~h}$ and then whole cell lysates were prepared. Western blot analyses were performed using adequate specific antibodies. D. Cells were transfected as described above and then cellular fractionation was performed. Western blot analysis was performed to determine the localization of NF-kB (p65) and $\alpha$-tubulin and lamin B was checked for cytoplasmic and nuclear markers, respectively. E-F. Cells were transfected with control (CTRL) or TAK1-specific siRNA and then the levels of TAK1 and phosphorylated IKK $\alpha / \beta$ and NF- $\kappa$ B were assessed by Western blot analysis. The level of miR-1908-5p was determined by RT-qPCR analysis. G-H. The protein and mRNA expression levels of NF- $\kappa \mathrm{B}$ target genes including HIF1- $\alpha$, cyclin D1, and slug, were determined by western blot and RT-qPCR analysis, respectively. All experiments are performed more than three times and data represent the mean \pm S.D. Asterisk $(*)$ indicates statistical significance of $\mathrm{p}<0.05$ as determined by Student's t-test. 
miR-1908-5p suppresses NKIRAS2 expression by directly binding to the 3'UTR of its mRNA

By screening miR-1908-5p target genes, we paid attention to NKIRAS2, a negative regulator of the NF- $\mathrm{KB}$ pathway. First, we checked the effect of miR-1908-5p on the expression of NKIRAS2 in U2OS cells. Overexpression of miR-1908-5p decreased the expression of NKIRAS2 and NKIRAS2 mRNA (Figures 5A and 5B, respectively).
To test whether miR-1908-5p directly binds to NKIRAS2 mRNA, Ago2 immunoprecipitation (IP) was performed using an Ago2-specific antibody (Figure 5C). Compared to the control, NKIRAS2 mRNA was more enriched in Ago2 IP materials due to overexpression of miR-1908-5p, indicating that the miR-1908-5p-loaded RISC complex interacts with NKIRAS2 mRNA. To verify the interaction between miR-1908-5p and NKIRAS2 mRNA, luciferase vectors containing wild-type and mutated sequences for
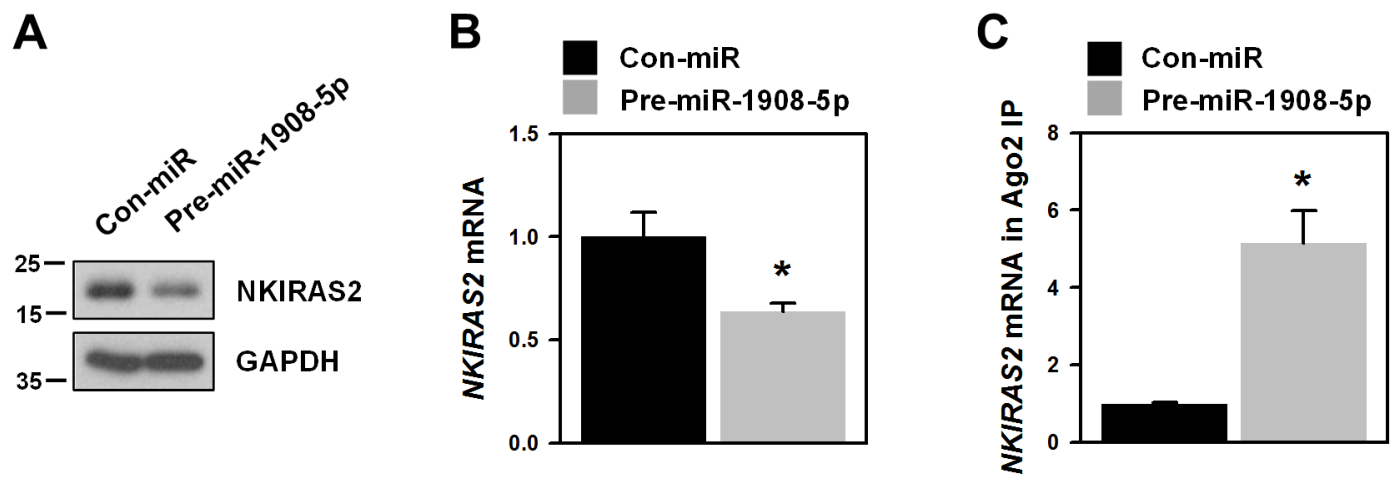

D

Human NKIRAS2 3'UTR length: $1748 \mathrm{nts}$
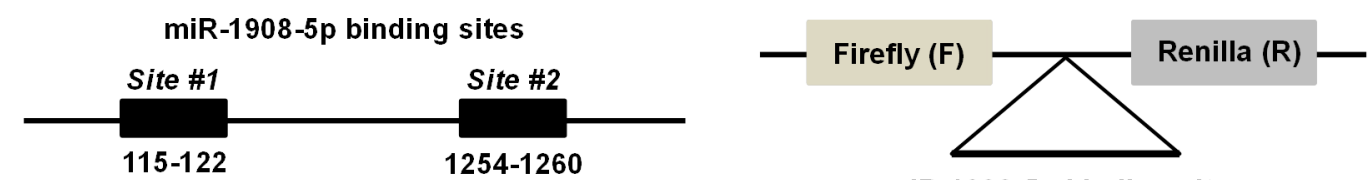

miR-1908-5p binding sites

Site \#1

5' CAGCCAGGGAGCUCCCCGCCA Wild type

3' CUGGUUAGCGGCAGGGGCGGC miR-1908-5p I I I

5' CAGCCAGGGAGCUCCGGCGCA Mutant type

Con-miR Pre-miR-1908-5p

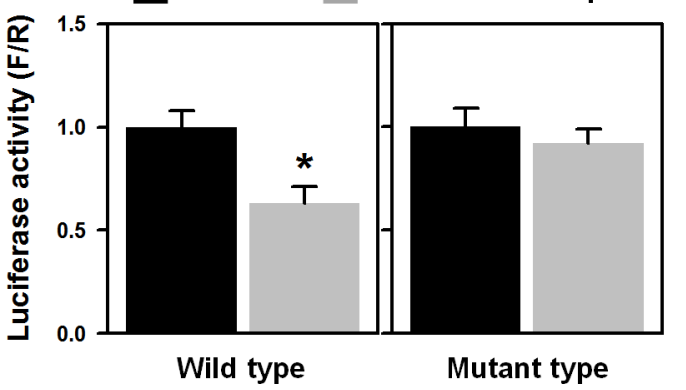

Site \#2

5' CGCUCAGUGCCCAGCCCGCCA Wild type

3` CUGGUUAGCGGCAGGGGCGGC miR-1908-5p 5' CGCUCAGUGCCCAGCGgCGCA Mutant type

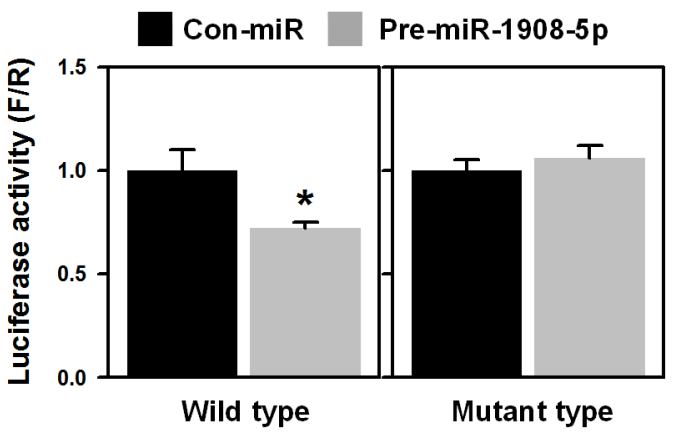

Figure 5: NKIRAS2 is a novel target of miR-1908-5p. A-B. U2OS cells were transfected with control (Con-miR) or pre-miR1908-5p for $48 \mathrm{~h}$ and then the level of NKIRAS2 protein and mRNA was assessed by western blot and RT-qPCR analysis, respectively. C. To check direct interaction between miRNA and target mRNA, Ago2 immunoprecipitation (IP) was performed using an Ago2-specifc antibody. Cytoplasmic lysates were prepared from control (CTRL) or pre-miR-1908-5p-transfectected cells and used for Ago2-IP. The mRNAs bound to miRNA-Ago2 were isolated and the level of NKIRAS2 mRNA was assessed by RT-qPCR analysis. D. To verify miR-1908-5p suppressed NKIRAS2, luciferase vectors harboring wild-type and mutated miR-1908-5p-binding sequences were manufactured. Luciferase activity was assessed as described in Materials and Methods. All experiments are performed more than three times and data represent the mean \pm S.D. Asterisk $(*)$ indicates statistical significance of $\mathrm{p}<0.05$ as determined by Student's t-test. 
miR-1908-5p were manufactured (Figure 5D). Since two binding sites are predicted, we manufactured two different reporter vectors. In both vectors, overexpression of miR$1908-5 p$ suppressed the luciferase activity. Conversely, it did not influence the luciferase activities of those containing mutated seed sequence for miR-1908-5p. Our results reveal that NKIRAS2 is a novel target gene for miR1908-5p.

\section{miR-1908-5p activates the transcriptional activity of NF-кB by suppressing NKIRAS2 expression}

Since NKIRAS2 is known to negatively regulate the NF- $\kappa$ B pathway by stabilizing I $\mathrm{B}-\beta$ and inhibiting nuclear localization of $\mathrm{NF}-\kappa \mathrm{B}(\mathrm{p} 65)$, we checked the level of I $\mathrm{B}-\beta$ and localization of NF- $\mathrm{B}$ (p65) in miR-
A
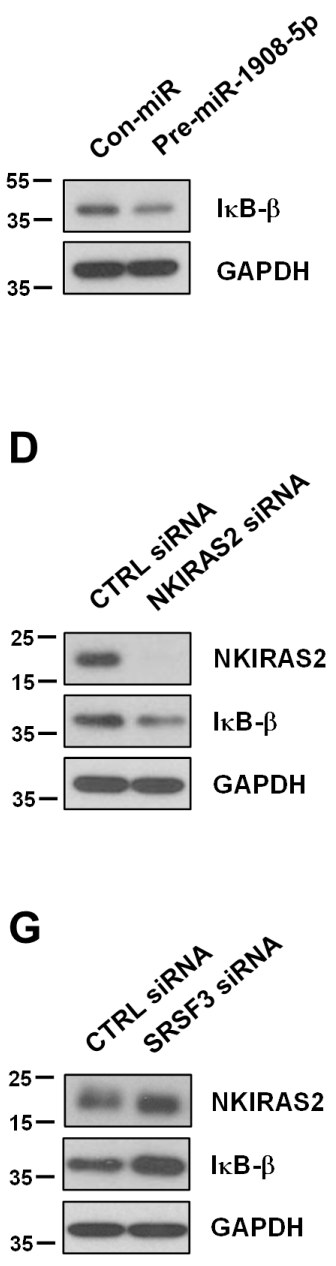

B

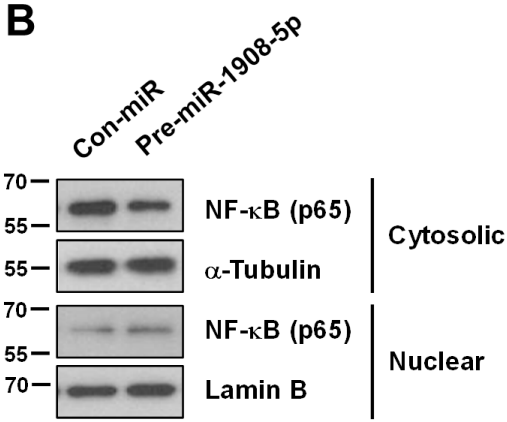

E

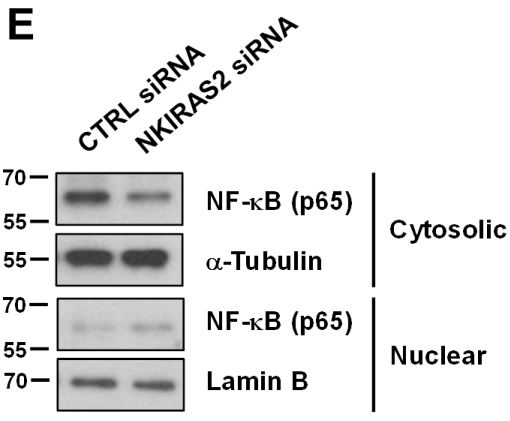

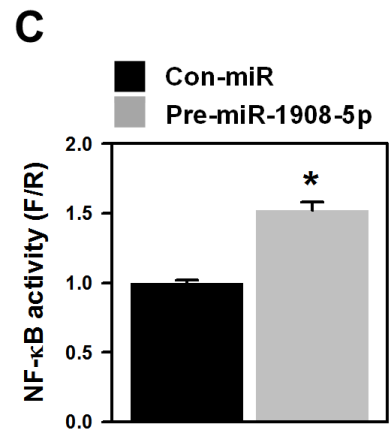

$\mathbf{F}$

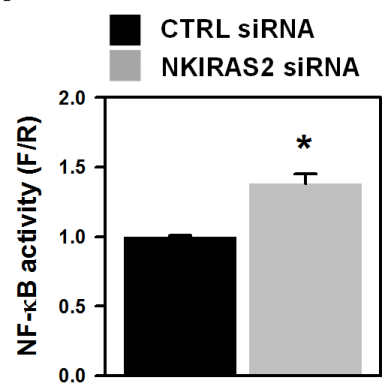

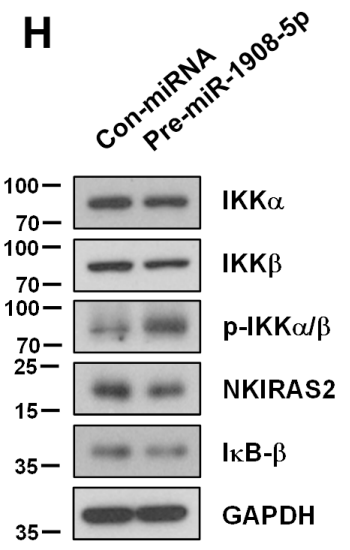

Figure 6: Downregulation of NKIRAS2 by miR-1908-5p is implicated in the oncogenic functions of SRSF3. A-F. U2OS cells were transfected with control (CTRL) or SRSF3-specific siRNA (A-C). In the case of miRNA, cells were transfected with control (Con-miR) or pre-miR-1908-5p (D-F). The level of IкB- $\beta$ was determined by western blot analysis (A and C). To assess the cytoplasmic and nuclear localization of NF-kB, cellular fractionation was performed and the level of NF- $\mathrm{kB}(\mathrm{p} 65$ ) in cytoplasmic and nuclear fraction was determined by western blot analysis (B and D). The level of $\alpha$-tubulin and lamin B was checked for cytoplasmic and nuclear markers, respectively. Transcriptional activity of $\mathrm{NF}-\mathrm{kB}$ was assessed by checking the luciferase activity as described in Materials and Methods (C and F). G. U2OS cells were transfected with control (CTRL) or SRSF3-specific siRNA and the level of NKIRAS2 and IkB- $\beta$ was determined by western blot analysis. H. U2OS cells were transfected with control (Con-miR) or pre-miR-1908-5p. After 48 h posttransfection, IKK $\alpha / \beta$ activation and expression of NKIRAS 2 and I $\kappa$ B $\beta$ were assessed by western blot analysis. 
1908-5p-overexpressed cells. As expected, the expression level of I B B- $\beta$ was decreased by overexpression of miR1908-5p (Figure 6A). In accordance with a diminished level of I $\kappa \mathrm{B}-\beta$, cytoplasmic localization of $\mathrm{NF}-\kappa \mathrm{B}$ (p65) was decreased (Figure 6B) and the transcriptional activity of NF- $\kappa \mathrm{B}$ was increased (Figure 6C). Knockdown of NKIRAS2 similarly decreased the level of $\mathrm{I} \kappa \mathrm{B}-\beta$ and cytoplasmic NF- $\kappa \mathrm{B}$ (p65), and activated the transcriptional activity of NF- $\kappa \mathrm{B}$ (Figures $6 \mathrm{D}, 6 \mathrm{E}$, and $6 \mathrm{~F}$, respectively). These results indicate that the miR-1908$5 \mathrm{p}$-mediated suppression of NKIRAS2 expression induces transactivation of NF- $\kappa$ B by destabilizing I $\kappa \mathrm{B}-\beta$ and inducing nuclear localization of NF- $\kappa \mathrm{B}$ (p65). Following transfection with SRSF3 siRNA or pre-miR-1908-5p, the expression level of NKIRAS 2 and I $\mathrm{B}-\beta$ was assessed by western blot analysis. In SRSF3-silenced cells, NKIRAS2 and I $\mathrm{B}-\beta$ were upregulated as compared to control, which is partly resulted from decreased expression of miR-19085p (Figure 6G). Conversely, transfection with miRNA mimic (pre-miR-1908-5p) activated IKK $\alpha / \beta$ and NF- $\kappa B$ by inducing their phosphorylation. It also suppressed the expression of NKIRAS2 and thus downregulated IкB- $\beta$, which indicating that miR-1908-5p is able to activate $\mathrm{NF}-\kappa \mathrm{B}$ pathway (Figure $6 \mathrm{H}$ ).

\section{miR-1908-5p-mediated downregulation of NKIRAS2 is involved in the oncogenic functions of SRSF3}

Next, we checked the role of the miR-1908-5p/ NKIRAS $2 / \mathrm{I} \kappa \mathrm{B}-\beta$ axis on the oncogenic functions of SRSF3. As with the knockdown of SRSF3, anchoragedependent or anchorage-independent cellular proliferation were enhanced by both overexpression of miR-1908$5 \mathrm{p}$ and knockdown of NKIRAS2 (Figure 7A and 7B). Invasive and migratory abilities were also potentiated by knockdown of NKIRAS2 (Figures 7C and 7D, respectively). These results suggest that miR-1908-5pelicited suppression of NKIRAS2 is responsible for the oncogenic function of SRSF3.

From the above results, we concluded that SRSF3 plays a critical role in cancer progression and confers malignant phenotypes on osteosarcoma U2OS cells. It was also found that miR-1908-5p, an NF- $\mathrm{BB}$-dependent
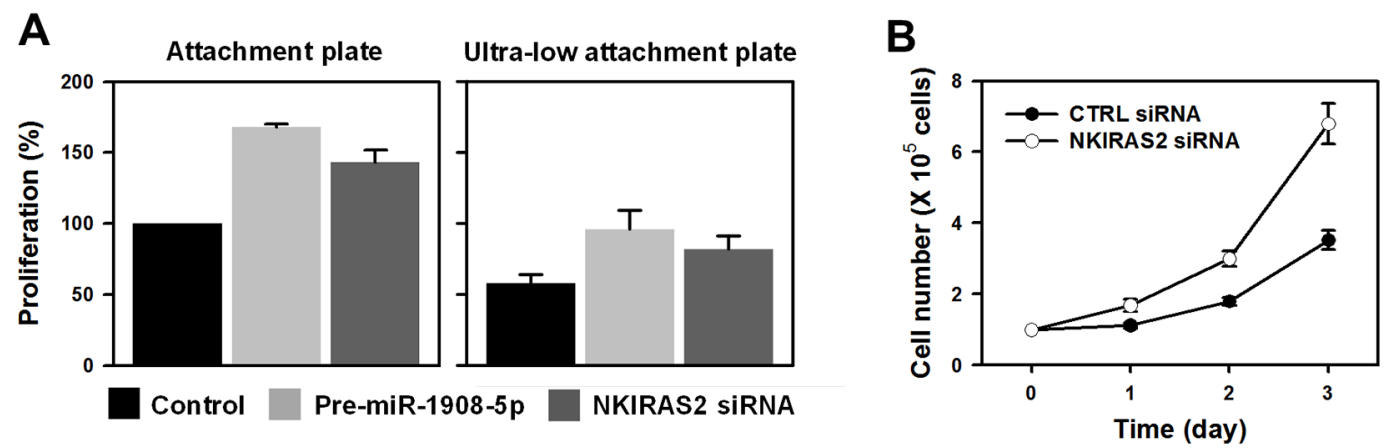

C

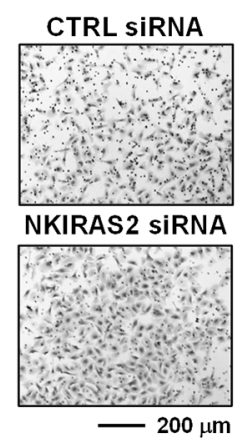

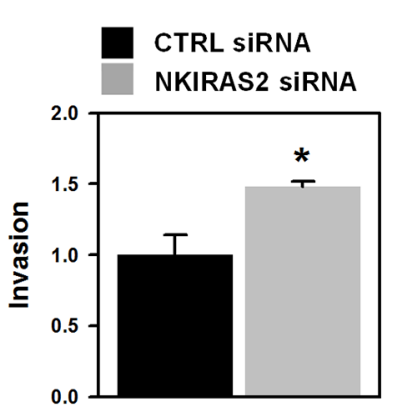

D
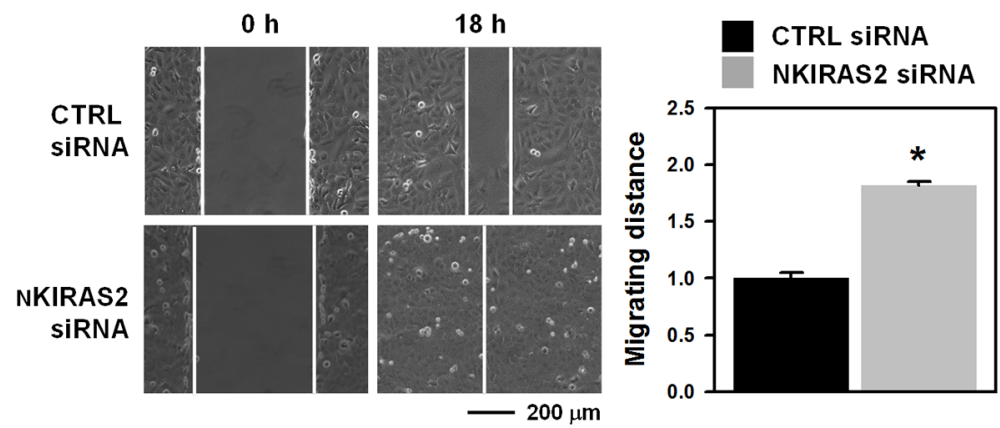

Figure 7: Downregulation of NKIRAS2 by miR-1908-5p is implicated in the oncogenic functions of SRSF3. A. To check the effect of miR-1908-5p/NKIRAS2 on the anchorage-independent proliferation, U2OS cells were transfected with pre-miR-1908-5p or NKIRAS2-specific siRNA for $24 \mathrm{~h}$. Equal numbers of transfected cells were plated in attachment or ultra-low attachment plates. After $48 \mathrm{~h}$ post-incubation, cellular viability was calculated by the MTS assay. B-D. U2OS cells were transfected with control (CTRL) or NKIRAS2specific siRNA for $48 \mathrm{~h}$. Cellular proliferation was calculated by counting viable cells every $24 \mathrm{~h}$ (B). Invasive and migratory abilities were assessed by Matrigel invasion (C) and wound healing assays (D), respectively. All experiments are performed more than three times and data represent the mean \pm S.D. Asterisk $\left(^{*}\right)$ indicates statistical significance of $p<0.05$ as determined by Student's t-test. 
oncogenic miRNA, is involved in oncogenic functions of SRSF3 and suppresses NKIRAS2 that potentiates the SRSF3-mediated transactivation of NF- $\kappa \mathrm{B}$. In conclusion, our results suggest that the SRSF3/miR-1908-5p/ NKIRAS2 axis is closely associated with the oncogenic functions of SRSF3 and is a promising target for cancer treatment. We proposed the molecular mechanism underlying oncogenic function of SRSF3 through NF- $\kappa \mathrm{B} /$ miR-1908-5p/NKIRAS2 axis (illustrated in Figure 8).

\section{DISCUSSION}

Emerging evidence has established that abnormal expression of SR proteins play a critical role in cancer progression mainly by affecting alternative splicing patterns of key transcripts. SR family proteins consist of 12 identified members in human and are characterized with RNA-binding domains and downstream RS domains [2]. The biological relevance of SR proteins is supported by researches proving that aberrant alternative splicing is closely linked with cancer progression. As an oncogene, SRSF3 plays a critical role in cell proliferation by promoting the G2/M transition and preventing apoptotic cell death [4]. In addition to its functions in alternative splicing process, SRSF3 is reported to control the mRNA translation [18] and transcription termination [19]. Several reports demonstrate that SRSF3 is an adapter protein for TAP-dependent export of mRNA [20-22] and is also involved in mRNA adenylation and therefore influences pre-mRNA processing [23].

Through genome-wide analyses of SRSF3 target genes, SRSF3 is reported to significantly influence the expression of a subset of oncogenic or tumor suppressive miRNAs [15]. Given the knowledge that pri-miRNAs are generally transcribed by RNA polymerase II and most miRNAs are located in the intron regions of genes, their expression levels are dependent on splicing events [24]. SRSF3 can regulate the expression of miRNA by controlling transcriptional or splicing events. In addition to its functions at the post-transcriptional level, SRSF3 is also known to influence miRNA biogenesis processes by regulating the Drosha-mediated cleavage of pri-miRNA [25]. In

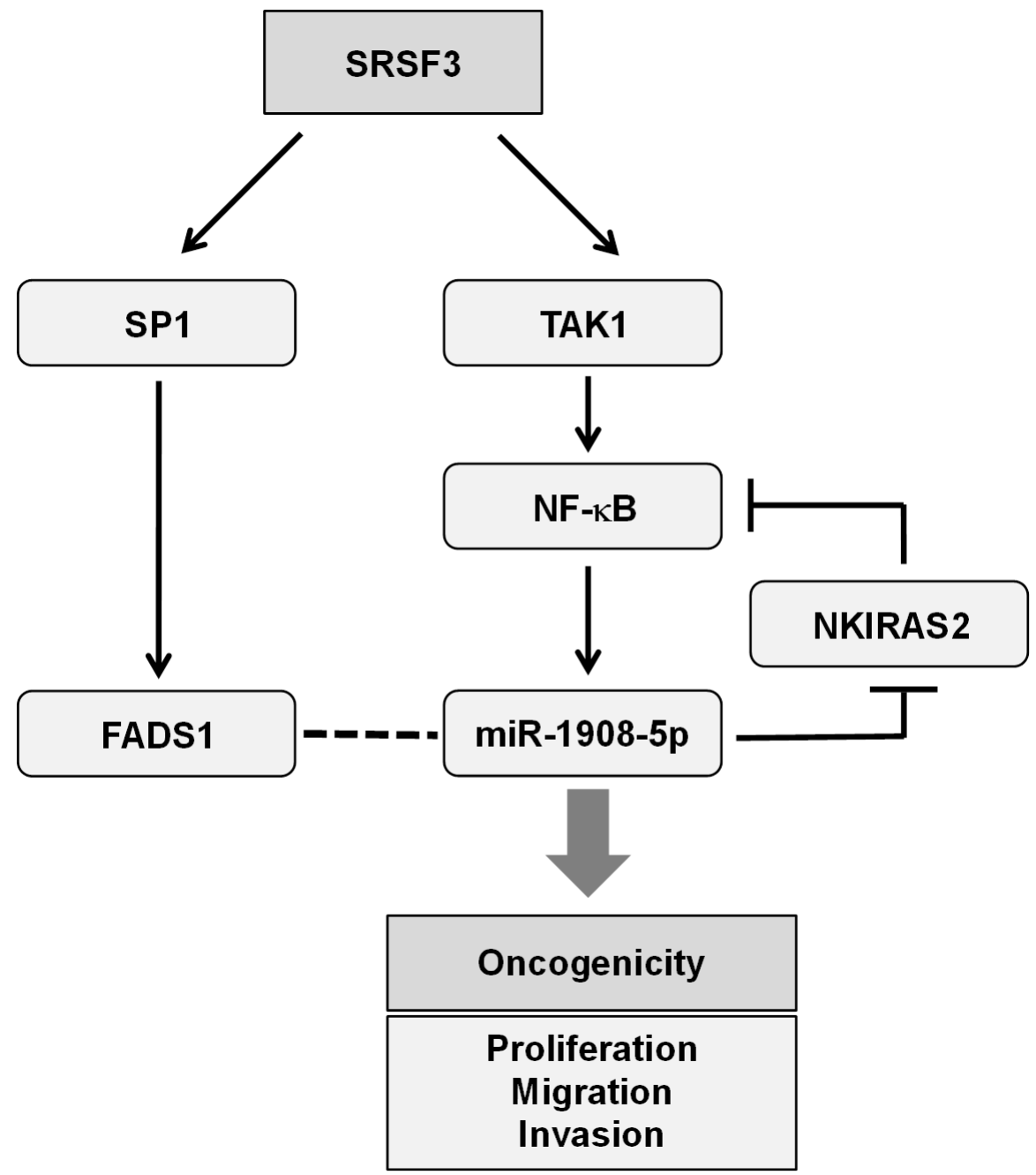

Figure 8: Proposed model for the role of miR-1908-5p in the oncogenic function of SRSF3. 
this report, SRSF3 was found to regulate the expression of miR-1908 and its host gene FADS1. However, knockdown of FADS1 did not influence the expression of miR-1908, indicating that these two SRSF3 targets are not closely associated. Moreover, FADS1 was not implicated in the oncogenic functions of SRSF3 either.

Most intronic miRNAs are known to be transcribed together with their host genes [26]. However, the level of several miRNAs are not correlated with the expression of its host gene, indicating that miRNAs could have their own transcriptional factors [27]. By investigating the oncogenic function of SRSF3, we previously observed that SRSF3 regulates a wide range of genes associated with cancer progression [9]. Among them, FADS1 is a fascinating target gene since it contains the sequence of miR-1908 in its intron. miR-1908 is an intronic miRNA encoded in the intron of FADS1 and is highly expressed in mature human adipocytes. It was recently reported that two putative binding sites of $\mathrm{NF}-\kappa \mathrm{B}$ are located in the promoter region of miR-1908 [16]. These findings suggest that miR-1908 is regulated by its own transcription factor, NF- $\kappa \mathrm{B}$. It was also observed that knockdown of SRSF3 inhibited the activation of IKK $\alpha$ and IKK $\beta$ and thus diminished the level of phosphorylated NF- $\kappa \mathrm{B}$ (p65), which resulted in the cytosolic retention of NF- $\kappa \mathrm{B}$ (p65). From these data, we demonstrate that SRSF3 is able to regulate the expression of miR-1908 by activating the NF- $\mathrm{KB}$ pathway.

The NF- $\kappa$ B pathway is known to be closely associated with cancer development and progression. By interacting with inhibitors of NF- $\kappa \mathrm{B}$ (I $\kappa \mathrm{Bs}$ such as $\mathrm{I} \kappa \mathrm{B} \alpha$, $\mathrm{I} \kappa \mathrm{B} \beta$, and $\mathrm{I} \kappa \mathrm{B} \varepsilon), \mathrm{NF}-\kappa \mathrm{B}$ dimers are sequestered in the cytoplasm and thus transcriptionally inactive in most types of cells [28]. During the activation of NF- $\kappa B$ signaling,

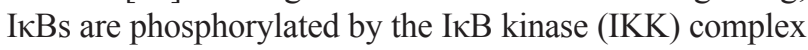
(two catalytic subunits, IKK $\alpha$ and IKK $\beta$, and one regulatory subunit, IKK $\gamma$ ) and are degraded through the ubiquitinproteasome pathway. Upon degradation of $\mathrm{I} \kappa \mathrm{B}, \mathrm{NF}-\kappa \mathrm{B}$ dimers translocate into the nucleus where they activate transcription of their target genes that are involved in cancer progression. Aberrant $\mathrm{NF}-\kappa \mathrm{B}$ activation is tightly associated with malignant conversion of premalignant cells and the production of cytokines, growth factors, proteases, and angiogenic factors in environmental cells $[29,30]$. Constitutively active $\mathrm{NF}-\kappa \mathrm{B}$ is also implicated in the gain of resistance against chemotherapeutics and radiation. NKIRAS2 is known as a potent regulator of the $\mathrm{NF}-\kappa \mathrm{B}$ pathway by preventing the degradation of $\mathrm{I} \kappa \mathrm{B}$, which is responsible for increased protein stability of IкB. In addition to transcriptional activation of its target genes, the NF- $\kappa \mathrm{B}$ pathway also plays central roles in cancer progression through upregulation of miRNAs. Abnormal activation of NF- $\kappa \mathrm{B}$ is closely implicated in biogenesis of cancer-associated miRNAs such as miR-146a [31, 32], miR-125b [32], miR-155 [33], miR-21 [34], miR-301a [35], miR-9 [36], miR-199a [37], and miR-15/16 cluster [38].
NKIRAS2 is an atypical Ras-like protein that functions as a negative regulator of the NF- $\mathrm{B}$ pathway by hindering the phosphorylation-dependent degradation of NF- $\kappa \mathrm{B}$ inhibitor beta (NFKBIB, also termed I $\kappa \mathrm{B}-\beta$ ). Therefore, NKIRAS2 inhibits the transactivation of NF$\kappa \mathrm{B}$ by stabilizing I $\mathrm{IB}-\beta$ and thus preventing the nuclear translocation of NF- $\kappa B$ (p65). We observed here that miR1908-5p suppressed the expression of NKIRAS2 through direct binding to the 3'UTR of its mRNA, which indicates that NKIRAS2 is a novel target of miR-1908-5p. Taken together, SRSF3 confers malignant phenotypes on cancer cells by upregulating the NF- $\kappa B-d e p e n d e n t ~ m i R-1908-5 p$ expression and its oncogenic functions are enforced by positive feedback regulation of the NF- $\kappa$ B pathway via the SRSF3/miR-1908-5p/NKIRAS2 axis.

\section{MATERIALS AND METHODS}

\section{Cell culture and transfection}

Human osteosarcoma U2OS cells were purchased from Korean Cell Line Bank (Republic of Korea) and maintained in Dulbecco's modified Eagles medium (high glucose, Hyclone, GE Healthcare, Little Chalfont, UK) supplemented with $10 \%$ fetal bovine serum and 1\% antibiotic-antimycotic solution (Gibco-BRL, Thermo Fisher Scientific, Waltham, MA, USA). U2OS cells were authenticated through short tandem repeat profiling.

For siRNA transfection, U2OS cells were plated at adequate density and transfected with the indicated siRNAs or control (CTRL) siRNA using Lipofectamine2000 (Invitrogen, Thermo Scientific) according to the manufacturer's protocol. The siRNAs for SRSF3 (GAGUGGAACUGUCGAAUGG) and NKIRAS2 (CGUCCUGGUCUAUAGCACA) were synthesized by Bioneer (Daejeon, Republic of Korea). FADS1-targeting (sc-96474) and Sp1-targeting siRNA (sc29487) were purchased from Santa Cruz Biotechnology (Santa Cruz, CA, USA). For overexpression of miRNA, miRNA-1908-5p mimic was purchased from GenePharma (Shanghai, China) and introduced into cells using Lipofectamine2000 (Invitrogen). Transfection of miRNA mimic sufficiently increased the level of miR-1908-5p (more than 1,000-fold increase).

\section{Cell proliferation and clonogenicity}

Transfected cells were resuspended into 12-well plates and the proliferation rate was determined by counting viable cells at every $24 \mathrm{~h}$. For the colony forming assay, an equal number of transfected cells (300 viable cells) were plated in 6-well plates and maintained in medium supplemented with serum. Two weeks later, the colonies were fixed with $4 \%$ paraformaldehyde and stained with crystal violet solution. Clonogenicity was assessed by counting the numbers of colonies. 


\section{Determination of metastatic potential}

Invasiveness was assessed using a $\mathrm{BD}$ Biocoat ${ }^{\mathrm{TM}}$ Matrigel ${ }^{\circledR}$ invasion chamber (BD Bioscience, San Jose, CA, USA). Equal cell numbers of transfected cells $\left(5 \times 10^{4}\right.$ cells $)$ were inoculated into the upper well and invasion was initiated by adding serum-containing medium into the lower well. After incubation for $24 \mathrm{~h}$, invading cells were stained with hematoxylin and eosin and photographed under a microscope. Invasive activities were determined by counting the number of invaded cells. For determination of migratory abilities, a wound healing assay was performed. Transfected cells were seeded into 12-well plates with high density and then a straight scratch was produced using a white pipette tip. After incubation for $16 \mathrm{~h}$, the migrated distance was calculated by AxioVision microscope software (ZEISS, Jena, Germany).

\section{RNA isolation and RT-qPCR}

After $48 \mathrm{~h}$ post-transfection, total RNA was isolated with TRIzol reagent (Ambion, USA) and was reverse transcribed using SuperScript III First-Strand Synthesis System (Invitrogen, USA). The product was used to perform real-time quantitative-PCR (RT-qPCR) amplification using the specific primers as follows: SRSF3 (forward, 5'-GCATCGTGATTCCTGTCCAT-3'; reverse, 5'-CGGAGTGGTCCATAGTAGCC-3'); FADS1 (forward, 5'-GCTACTTCACCTGGGACGAG-3'; reverse, 5'-GGTGAACTCGCTGATGTTGT-3'); pri-miR-1908 (forward, 5'-CAGACGAAACAGGCACCAAC-3'; reverse, 5'-CACATACGGACCAATCGCCG-3'); Sp1 (forward, 5'-GTGGAGGCAACATCATTGCTG-3'; reverse, 5'-CCACTGGTACATTGGTCACAT-3'); NKIRAS2 (forward, 5'-GAGAGCAGGTGCGTTTCTATG-3'; reverse, 5'-ACCAGGACGTAGCCATCAGT-3'); Cyclin D1 (forward, 5'-CCGTCCATGCGGAAGATC-3'; reverse, 5'-ATGGCCAGCGGGAAGAC-3'); Slug (forward, 5'-TGTTGCAGTGAGGGCAAGAA-3'; reverse, 5'-GACCCTGGTTGCTTCAAGGA-3'); HIF-1 $\alpha$ (forward, 5'-CACTACCACTGCCACCACTG-3'; reverse, 5'-TGGGTAGGAGATGGAGATGC-3'); and GAPDH (forward, 5'-TGCACCACCAACTGCTTAGC-3'; reverse, 5'-GGCATGGACTGTGGTCATGAG-3'). For detection of miRNA, TaqMan ${ }^{\circledR}$ gene expression assays were performed using hsa-1908-5p-specific and has-miR-1908-3p specific primers (Applied Biosystems, USA).

\section{Preparation of cytoplasmic and nuclear extracts}

For isolation of cytosolic and nuclear fractions, cells were harvested and lysed with digitonin-containing RSB buffer. After centrifugation at 2,000 $\times \mathrm{g}$ for $8 \mathrm{~min}$, the supernatant was transferred to a new tube (cytosolic extracts). The remaining pellet was washed four times with RSB buffer and lysed with RIAP buffer. Nuclear extracts (NE) were isolated by centrifugation at 13,200 rpm for $30 \mathrm{~min}$ [39].

\section{Western blot analysis}

Whole cell lysates were prepared using RIPA buffer containing phosphatase inhibitor and protease inhibitor cocktail (Roche, Basel, Switzerland). Following standard western blotting procedure, proteins were blotted onto PVDF membrane (Millipore, Bilerica, MA, USA) and recognized by the following antibodies: SRSF3 (sc-13510), $\alpha$-tubulin (sc-69969), and lamin B (sc-6216) were from Santa Cruz Biotechnology; Sp1 (\#5931), IKK $\alpha$ (\#2682), IKK $\beta$ (\#2370); p-IKK $\alpha / \beta$

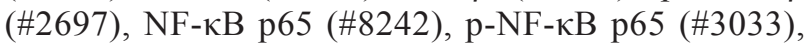
Cyclin D1 (\#2926), and Slug (\#9585) were from Cell Signaling Technology (Danvers, MA, USA); IкB- $\beta$ (A301-828A) was from Bethyl Laboratories (Montgomery, TX, USA); HIF1- $\alpha$ (610958) was from BD Bioscience; NKIRAS2 (ab57303), GAPDH (ab8245), and $\beta$-actin (ab8226) were from Abcam (Cambridge, UK).

\section{Luciferase reporter assay}

To assess the transactivation of NF- $\mathrm{KB}$, a pGL4.32 [luc2P/NF-kB-RE/Hygro] vector (E849A; Promega, Madison, WI, USA) containing an NF- $\mathrm{KB}$ responsive element was used. U2OS cells were transfected with siRNA or miRNA for $24 \mathrm{~h}$ and then the firefly reporter vectors were transfected along with renilla expressing vector (E2231; Promega).

For validation of miRNA recognition elements, the luciferase constructs containing the binding sites of miR-1908-5p were manufactured using a pmirGLO vector (E133A; Promega). Since two positions are predicted for the miR-1908-5p binding site in the 3'UTR of NKIRAS2 mRNA, we prepared two individual vectors containing wild-type or mutant sequences of miR-1908-5p. In order to check the effect of miR-1908-5p on NKIRAS2 expression, U2OS cells were seeded in 24-well plates with around 50\% confluency and transfected with the indicated siRNA or miRNA. At $24 \mathrm{~h}$ post-transfection, cells were transfected with either a pmirGLO blank vector or pmirGLO-NKIRAS2/3'UTR vector (willd-type or mutant). The luciferase activity was measured $24 \mathrm{~h}$ later using Dual-Glo Luciferase Assay System (E2940; Promega) on a GloMax ${ }^{\circledR} 20 / 20$ Luminometer (Promega).

\section{Ago2 immunoprecipitation}

To check the interaction between miRNA and NKIRAS2 mRNA, immunoprecipitation (IP) with Ago2 was performed. Briefly, Ago2 antibody (SigmaAldrich, St. Louis, MO, USA) and Dynabeads Protein $\mathrm{G}$ (Invitrogen) were incubated at $4{ }^{\circ} \mathrm{C}$ with rotation a day 
prior to the experiment. Cytoplasmic lysates of transfected cells were prepared with PEB buffer and incubated with Ago 2 antibody-coated beads for $3 \mathrm{~h}$. RNAs in the Ago2 IP materials were isolated and then the level of NKIRAS2 mRNA was determined by RT-qPCR [39].

\section{CONFLICTS OF INTEREST}

The authors declare no competing financial interests.

\section{GRANT SUPPORT}

This study was supported by a grant from the Mid-career Research Program through the National Research Foundation (NRF) funded by the Ministry of Science, ICT, and Future Planning, Republic of Korea (2014R1A2A1A11053130), by a grant of the Korea Health Technology R\&D Project through the Korea Health Industry Development Institute (KHIDI), funded by the Ministry of Health \& Welfare, Republic of Korea (HI14C3418), and by a grant (MRC-2015-009070) from the National Research Foundation of Korea funded by the Korea government (MSIP).

\section{REFERENCES}

1. Guo J, Jia J, Jia R. PTBP1 and PTBP2 impaired autoregulation of SRSF3 in cancer cells. Sci Rep. 2015; 5: 14548. doi: 10.1038/srep14548.

2. Corbo C, Orru S, Salvatore F. SRp20: an overview of its role in human diseases. Biochem Biophys Res Commun. 2013; 436: 1-5. doi: 10.1016/j.bbrc.2013.05.027.

3. Long JC, Caceres JF. The SR protein family of splicing factors: master regulators of gene expression. Biochem J. 2009; 417: 15-27. doi: 10.1042/BJ20081501.

4. Jia R, Li C, McCoy JP, Deng CX, Zheng ZM. SRp20 is a proto-oncogene critical for cell proliferation and tumor induction and maintenance. Int J Biol Sci. 2010; 6: 806-26.

5. Zahler AM, Lane WS, Stolk JA, Roth MB. SR proteins: a conserved family of pre-mRNA splicing factors. Genes Dev. 1992; 6: 837-47.

6. He X, Arslan AD, Pool MD, Ho TT, Darcy KM, Coon JS, Beck WT. Knockdown of splicing factor SRp20 causes apoptosis in ovarian cancer cells and its expression is associated with malignancy of epithelial ovarian cancer. Oncogene. 2011; 30: 356-65. doi: 10.1038/onc.2010.426.

7. Kurokawa K, Akaike Y, Masuda K, Kuwano Y, Nishida K, Yamagishi N, Kajita K, Tanahashi T, Rokutan K. Downregulation of serine/arginine-rich splicing factor 3 induces G1 cell cycle arrest and apoptosis in colon cancer cells. Oncogene. 2014; 33: 1407-17. doi: 10.1038/ onc.2013.86.

8. Jang HN, Lee M, Loh TJ, Choi SW, Oh HK, Moon H, Cho S, Hong SE, Kim do H, Sheng Z, Green MR, Park D, Zheng
$\mathrm{X}$, et al. Exon 9 skipping of apoptotic caspase-2 pre-mRNA is promoted by SRSF3 through interaction with exon 8 . Biochim Biophys Acta. 2014; 1839: 25-32. doi: 10.1016/j. bbagrm.2013.11.006.

9. Kim J, Park RY, Chen JK, Kim J, Jeong S, Ohn T. Splicing factor SRSF3 represses the translation of programmed cell death 4 mRNA by associating with the 5'-UTR region. Cell Death Differ. 2014; 21: 481-90. doi: 10.1038/cdd.2013.171.

10. Krol J, Loedige I, Filipowicz W. The widespread regulation of microRNA biogenesis, function and decay. Nat Rev Genet. 2010; 11: 597-610. doi: 10.1038/nrg2843.

11. Jansson MD, Lund AH. MicroRNA and cancer. Mol Oncol. 2012; 6: 590-610. doi: 10.1016/j.molonc.2012.09.006.

12. Karni R, de Stanchina E, Lowe SW, Sinha R, Mu D, Krainer $\mathrm{AR}$. The gene encoding the splicing factor SF2/ASF is a proto-oncogene. Nat Struct Mol Biol. 2007; 14: 185-93. doi: 10.1038/nsmb1209.

13. Jensen MA, Wilkinson JE, Krainer AR. Splicing factor SRSF6 promotes hyperplasia of sensitized skin. Nat Struct Mol Biol. 2014; 21: 189-97. doi: 10.1038/nsmb.2756.

14. Cohen-Eliav M, Golan-Gerstl R, Siegfried Z, Andersen CL, Thorsen K, Orntoft TF, Mu D, Karni R. The splicing factor SRSF6 is amplified and is an oncoprotein in lung and colon cancers. J Pathol. 2013; 229: 630-9. doi: 10.1002/path.4129.

15. Ajiro M, Jia R, Yang Y, Zhu J, Zheng ZM. A genome landscape of SRSF3-regulated splicing events and gene expression in human osteosarcoma U2OS cells. Nucleic Acids Res. 2016; 44: 1854-70. doi: 10.1093/nar/ gkv1500.

16. Kuang Q, Li J, You L, Shi C, Ji C, Guo X, Xu M, Ni Y. Identification and characterization of NF-kappaB binding sites in human miR-1908 promoter. Biomed Pharmacother. 2015; 74: 158-63. doi: 10.1016/j.biopha.2015.08.018.

17. Freudlsperger C, Bian Y, Contag Wise S, Burnett J, Coupar J, Yang X, Chen Z, Van Waes C. TGF-beta and NF-kappaB signal pathway cross-talk is mediated through TAK1 and SMAD7 in a subset of head and neck cancers. Oncogene. 2013; 32: 1549-59. doi: 10.1038/onc.2012.171.

18. Bedard KM, Daijogo S, Semler BL. A nucleo-cytoplasmic SR protein functions in viral IRES-mediated translation initiation. EMBO J. 2007; 26: 459-67. doi: 10.1038/ sj.emboj.7601494.

19. Cui M, Allen MA, Larsen A, Macmorris M, Han M, Blumenthal T. Genes involved in pre-mRNA 3'-end formation and transcription termination revealed by a lin15 operon Muv suppressor screen. Proc Natl Acad Sci U S A. 2008; 105: 16665-70. doi: 10.1073/pnas.0807104105.

20. Huang Y, Gattoni R, Stevenin J, Steitz JA. SR splicing factors serve as adapter proteins for TAP-dependent mRNA export. Mol Cell. 2003; 11: 837-43.

21. Hautbergue GM, Hung ML, Golovanov AP, Lian LY, Wilson SA. Mutually exclusive interactions drive handover of mRNA from export adaptors to TAP. Proc Natl Acad Sci U S A. 2008; 105: 5154-9. doi: 10.1073/pnas.0709167105. 
22. Escudero-Paunetto L, Li L, Hernandez FP, Sandri-Goldin RM. SR proteins SRp20 and 9G8 contribute to efficient export of herpes simplex virus 1 mRNAs. Virology. 2010; 401: 155-64. doi: 10.1016/j.virol.2010.02.023.

23. Lou H, Neugebauer KM, Gagel RF, Berget SM. Regulation of alternative polyadenylation by U1 snRNPs and SRp20. Mol Cell Biol. 1998; 18: 4977-85.

24. Du P, Wang L, Sliz P, Gregory RI. A Biogenesis Step Upstream of Microprocessor Controls miR-17 approximately 92 Expression. Cell. 2015; 162: 885-99. doi: 10.1016/j.cell.2015.07.008.

25. Auyeung VC, Ulitsky I, McGeary SE, Bartel DP. Beyond secondary structure: primary-sequence determinants license pri-miRNA hairpins for processing. Cell. 2013; 152: 84458. doi: 10.1016/j.cell.2013.01.031.

26. Ronchetti D, Lionetti M, Mosca L, Agnelli L, Andronache A, Fabris S, Deliliers GL, Neri A. An integrative genomic approach reveals coordinated expression of intronic miR335, miR-342, and miR-561 with deregulated host genes in multiple myeloma. BMC Med Genomics. 2008; 1: 37. doi: 10.1186/1755-8794-1-37.

27. Monteys AM, Spengler RM, Wan J, Tecedor L, Lennox KA, Xing Y, Davidson BL. Structure and activity of putative intronic miRNA promoters. RNA. 2010; 16: 495-505. doi: 10.1261/rna.1731910.

28. Inoue J, Gohda J, Akiyama T, Semba K. NF-kappaB activation in development and progression of cancer. Cancer Sci. 2007; 98: 268-74. doi: 10.1111/j.1349-7006.2007.00389.x.

29. Greten FR, Eckmann L, Greten TF, Park JM, Li ZW, Egan LJ, Kagnoff MF, Karin M. IKKbeta links inflammation and tumorigenesis in a mouse model of colitis-associated cancer. Cell. 2004; 118: 285-96. doi: 10.1016/j.cell.2004.07.013.

30. Pikarsky E, Porat RM, Stein I, Abramovitch R, Amit S, Kasem S, Gutkovich-Pyest E, Urieli-Shoval S, Galun E, Ben-Neriah Y. NF-kappaB functions as a tumour promoter in inflammation-associated cancer. Nature. 2004; 431: $461-$ 6. doi: 10.1038/nature02924.

31. Taganov KD, Boldin MP, Chang KJ, Baltimore D. NF-kappaB-dependent induction of microRNA miR-146, an inhibitor targeted to signaling proteins of innate immune responses. Proc Natl Acad Sci U S A. 2006; 103: 12481-6. doi: 10.1073/pnas.0605298103.
32. Pogue AI, Percy ME, Cui JG, Li YY, Bhattacharjee S, Hill JM, Kruck TP, Zhao Y, Lukiw WJ. Up-regulation of NF-kBsensitive miRNA-125b and miRNA-146a in metal sulfatestressed human astroglial (HAG) primary cell cultures. J Inorg Biochem. 2011; 105: 1434-7. doi: 10.1016/j. jinorgbio.2011.05.012.

33. O'Connell RM, Taganov KD, Boldin MP, Cheng G, Baltimore D. MicroRNA-155 is induced during the macrophage inflammatory response. Proc Natl Acad Sci U S A. 2007; 104: 1604-9. doi: 10.1073/pnas.0610731104.

34. Sheedy FJ, Palsson-McDermott E, Hennessy EJ, Martin C, O’Leary JJ, Ruan Q, Johnson DS, Chen Y, O’Neill LA. Negative regulation of TLR4 via targeting of the proinflammatory tumor suppressor PDCD4 by the microRNA miR-21. Nat Immunol. 2010; 11: 141-7. doi: 10.1038/ni.1828.

35. Lu Z, Li Y, Takwi A, Li B, Zhang J, Conklin DJ, Young KH, Martin R, Li Y. miR-301a as an NF-kappaB activator in pancreatic cancer cells. EMBO J. 2011; 30: 57-67. doi: 10.1038/emboj.2010.296.

36. Bazzoni F, Rossato M, Fabbri M, Gaudiosi D, Mirolo M, Mori L, Tamassia N, Mantovani A, Cassatella MA, Locati $\mathrm{M}$. Induction and regulatory function of miR-9 in human monocytes and neutrophils exposed to proinflammatory signals. Proc Natl Acad Sci U S A. 2009; 106: 5282-7. doi: 10.1073/pnas.0810909106.

37. Chen R, Alvero AB, Silasi DA, Kelly MG, Fest S, Visintin I, Leiser A, Schwartz PE, Rutherford T, Mor G. Regulation of IKKbeta by miR-199a affects NF-kappaB activity in ovarian cancer cells. Oncogene. 2008; 27: 4712-23. doi: 10.1038/onc.2008.112.

38. Li T, Morgan MJ, Choksi S, Zhang Y, Kim YS, Liu ZG. MicroRNAs modulate the noncanonical transcription factor NF-kappaB pathway by regulating expression of the kinase IKKalpha during macrophage differentiation. Nat Immunol. 2010; 11: 799-805. doi: 10.1038/ni.1918.

39. Lee H, Hwang SJ, Kim HR, Shin CH, Choi KH, Joung JG, Kim HH. Neurofibromatosis 2 (NF2) controls the invasiveness of glioblastoma through YAP-dependent expression of CYR61/CCN1 and miR-296-3p. Biochim Biophys Acta. 2016; 1859: 599-611. doi: 10.1016/j. bbagrm.2016.02.010. 